Sharif University of Technology
Scientia Iranica
SCIENTIA
IRAN Transactions B: Mechanical Engineering
www.scientiairanica.com

\title{
A reliable implicit difference scheme for treatments of fourth-order fractional sub-diffusion equation
}

\author{
K. Sayevand* and F. Arjang \\ Faculty of Mathematical Sciences, Malayer University, Malayer, P.O. Box 16846-13114, Iran.
}

Received 12 October 2015; received in revised form 17 December 2015; accepted 18 June 2016

\author{
KEYWORDS \\ Ji Huan He's \\ fractional derivative; \\ Convergence; \\ Finite difference \\ approximation; \\ Fractional differential \\ equations; \\ Stability.
}

\begin{abstract}
In this paper, a reliable implicit difference scheme is proposed to analyze the fractional fourth-order subdiffusion equation on a bounded domain. The time-fractional derivative operator is characterized in the Ji Huan He's sense, and the space derivative is approximated by the five-point centered formula. The numerical parameters, i.e. consistency, stability, and convergence analyses of the considered scheme, are proven.
\end{abstract}

(C) 2017 Sharif University of Technology. All rights reserved.

\section{Introduction}

The fractional calculus was invented over three centuries ago. Recently, the fractional calculus has become a very important topic to the development of promising analytical and numerical schemes (e.g., the homotopy perturbation method, the variational iteration method, finite difference approximation, etc.). It is noted that some new foundation in fractional calculus can be found in [1-19].

In recent decades, for modeling the anomalous subdiffusion equation, a theoretical framework has been examined based on the fractional calculus and the physics of continuous time random walk [20]. Anomalous subdiffusion process can appear in spatially disordered systems, fractal media, biological media with traps, porous media, turbulent fluids and plasmas, binding sites or macro-molecular crowding, etc. For more details, see [21-24].

Finding proper solutions to anomalous subdiffusion equations is a very important matter in the

*. Corresponding author. Tel./Fax: +98 8133340867

E-mail addresses: ksayehvand@malayeru.ac.ir (K.

Sayevand); fateme.arjang@stu.malayeru.ac.ir. (F. Arjang) theory of fractional calculus. In this paper, to present solutions to fractional subdiffusion equation, a finite difference approximation is investigated. As we know, this approach replaces the appropriate estimate for each derivative in the differential equations based on nodal values and estimates derivatives of a known function only by values of the function itself at these discrete points. We recall the time when fractional diffusion equations were scrutinized in both analytical and numerical frames by several authors (see for example [25-28]). But, according to fundamental role of this class of equations in science and engineering, we will talk about it again.

In the theory of fractional partial differential equations, we have the $2 n$-order subdiffusion equation as in the following (initial and boundary values) problem:

$$
\left\{\begin{array}{l}
\frac{\partial^{\alpha} \psi}{\partial t^{\alpha}}(x, t)+\beta(-\Delta)^{n} \psi(x, t)=g(x, t), \\
\text { in } \Omega \times[0, T], \quad 0<\alpha<1, \quad n \in N, \\
\Delta^{k} \psi(x, t)=0, \\
\text { on } \partial \Omega \times[0, T], \quad k=0,1, \cdots, n-1, \\
\psi(x, 0)=v(x), \\
\text { in } \Omega,
\end{array}\right.
$$


and it is very important in applications from both theoretical and numerical points of view. The data of the problem, $v$ and $g$, are sufficiently smooth functions, and $\beta$ is the fractional diffusion coefficient. For $x=$ $\left(x_{1}, \cdots, x_{d}\right), \Delta$ is the Laplacian defined by $\Delta \psi=$ $\sum_{j=1}^{d} \frac{\partial^{2} \psi}{\partial x_{j}^{2}}$, and $\Omega$ is a bounded domain in $d$-dimensional Euclidean space $R^{d}$ with boundary $\partial \Omega$ (see [8]).

The generalized equation in (Eq. (1)) is shown to be put on the propagation of stress waves in viscoelastic solids, presenting a power low creep of degree $p$ when $1<p<2$, and then $0<\alpha=2-p<1$ (for more details, see [8]). This generalization was applied to introduce a method to describe subdiffusive anomalous phenomena associated with mechanical behavior of certain materials [29].

In this article, based on the finite difference approximation, we devise an accurate and precise numerical scheme to solve the sub-diffusion equation defined in Eq. (1) for cases $d=1$ and $n=2$. For convenience, assume $\Omega=[a, b]$.

The paper is organized as follows. In Section 2, we use the modified finite difference formula for the time and space derivatives and obtain an approximate solution of Eq. (1). Section 3 is devoted to investigate some error analyses of the numerical approach, that is, we show that the proposed method is unconditionally stable and convergent. Section 4 contains a numerical example to illustrate the accuracy and robustness of the proposed finite difference approximation. The concluding remarks are collected in Section 5 .

\section{Analysis of the implicit difference method}

$t_{k}=k \tau, k=0,1,2, \cdots, N$, and $x_{i}=i h, i=$ $0,1,2, \cdots, M$, are denoted, where $h=\frac{L}{M}$ and $\tau=$ $\frac{T}{N}$ are space and time steps, respectively. For two independent variables, $x$ and $t,\left(x_{i}, t_{k}\right)$ is the coordinate of $(i, k)$ node of this mesh. Let $\psi\left(x_{i}, t_{k}\right)$ be the exact solution to the proposed equation at $\left(x_{i}, t_{k}\right)$, and application of the finite difference approximation, $\psi_{i}^{k}$, be the numerical approximation of it. We also suppose that:

$$
\triangle_{t} \psi\left(x_{i}, t_{k}\right)=\psi\left(x_{i}, t_{k+1}\right)-\psi\left(x_{i}, t_{k}\right)
$$

The fractional derivative (in time dimension) proposed here is the Ji Huan He's fractional derivative [1]. This derivative is defined as:

$$
\begin{aligned}
D_{t}^{\alpha} \psi(t)= & \frac{1}{\Gamma(m-\alpha)} \frac{d^{m}}{d t^{m}} \int_{t_{0}}^{t}(\tau-t)^{m-\alpha-1} \\
& \left(\psi_{0}(\tau)-\psi(\tau)\right) d \tau \\
m-1<\alpha<m &
\end{aligned}
$$

where $\alpha$ is the order of the derivative which can be real or complex. Prof. Ji Huan He in [1] showed that:

1. For a continuous and differentiable function, $\psi_{0}(t)$, the following relation holds:

$$
\begin{aligned}
\psi_{0}(t)= & \psi\left(t_{0}\right)+\left(t-t_{0}\right) \psi^{\prime}\left(t_{0}\right)+\frac{1}{2}\left(t-t_{0}\right)^{2} \psi^{\prime \prime}\left(t_{0}\right) \\
& +\cdots+\frac{1}{(m-1) !}\left(t-t_{0}\right)^{n-1} \psi^{(m-1)}\left(t_{0}\right)
\end{aligned}
$$

2. If $\psi_{0}(t)$ is continuous but not differentiable anywhere, Eq. (3) can be presented in the following form:

$$
\begin{aligned}
\psi_{0}(t)= & \psi\left(t_{0}\right)+\frac{\left(t-t_{0}\right)}{\Gamma(1+\alpha)} \psi^{(\alpha)}\left(t_{0}\right) \\
& +\frac{\left(t-t_{0}\right)^{2}}{\Gamma(1+2 \alpha)} \psi^{(2 \alpha)}\left(t_{0}\right)+\cdots \\
& +\frac{\left(t-t_{0}\right)^{m-1}}{\Gamma(1+(m-1) \alpha)} \psi^{((m-1) \alpha)}\left(t_{0}\right)
\end{aligned}
$$

3. Eq. (3) has the following property for continuous and differentiable functions:

$$
\begin{aligned}
D_{t}^{\alpha} \psi(t)= & \frac{1}{\Gamma(m-\alpha)} \frac{d^{m}}{d t^{m}} \int_{t_{0}}^{t}(\tau-t)^{m-\alpha-1} \\
& \left(\sum_{i=0}^{m-1} \frac{1}{i !}\left(\tau-t_{0}\right)^{i} \psi^{(i)}\left(t_{0}\right)-\psi(\tau)\right) d \tau
\end{aligned}
$$

4. Also, for continuous and non-differentiable functions, Eq. (3) can be equivalent to the following relation:

$$
\begin{aligned}
D_{t}^{\alpha} \psi(t)= & \frac{1}{\Gamma(m-\alpha)} \frac{d^{m}}{d t^{m}} \int_{t_{0}}^{t}(\tau-t)^{m-\alpha-1} \\
& \left(\sum_{i=0}^{m-1} \frac{\left(\tau-t_{0}\right)^{i}}{\Gamma(1+i \alpha)} \psi^{(i \alpha)}\left(t_{0}\right)-\psi(\tau)\right) d \tau
\end{aligned}
$$

We advise readers to see more scientific results of $\mathrm{Ji}$ Huan He's fractional derivative in [3,4], respectively.

Hereunder, for containing the discretized form of fractional derivative operator in time dimension, we use a backward finite difference approximation $\left(L_{1^{-}}\right.$ concept) as [27]:

$$
\frac{\partial^{\alpha} \psi\left(x_{i}, t_{k+1}\right)}{\partial t^{\alpha}} \approx \frac{\tau^{-\alpha}}{\Gamma(2-\alpha)} \sum_{j=0}^{k} b_{j} \triangle_{t} \psi\left(x_{i}, t_{k-j}\right)
$$

where $b_{j}=(j+1)^{1-\alpha}-j^{1-\alpha}, j=0,1, \cdots, N$, and the following relations hold: 
1. $b_{j}>0, j=1,2, \cdots$,

2. $b_{j}>b_{j+1}, j=1,2, \cdots$.

For the fourth-order fractional derivative in space dimension, we apply the centered finite difference concept at $t_{k+1}$ as follows:

$$
\begin{aligned}
\frac{\partial^{4} \psi\left(x_{i}, t_{k+1}\right)}{\partial x^{4}}=\frac{1}{h^{4}}\left[\psi_{i-2}^{k+1}-4 \psi_{i-1}^{k+1}+6 \psi_{i}^{k+1}\right. \\
\\
\left.\quad-4 \psi_{i+1}^{k+1}+\psi_{i+2}^{k+1}\right]+O\left(h^{2}\right) .
\end{aligned}
$$

Furthermore, we set these notations for simplicity:

$$
\left\{\begin{array}{l}
g_{i}^{k+1}=g\left(x_{i}, t_{k+1}\right)=g(i h,(k+1) \tau), \\
d_{i}^{k}=\tau^{\alpha} \Gamma(2-\alpha) g_{i}^{k} \\
r=r(i, k)=\frac{\tau^{\alpha} \Gamma(2-\alpha) \beta}{h^{4}}, \\
r_{1}=\tau^{\alpha} \Gamma(2-\alpha) .
\end{array}\right.
$$

In the present approximate scheme, to solve the problem, Expressions (8), (9), and (10) are substituted into Eq. (1). This operation leads to a recurrence relationship between successive time levels of related unknown element parameters $\psi_{i}^{k+1}(i=1,2, \cdots, M-$ $1, k=1,2, \cdots, N)$ that can be presented in the following form:

$$
\begin{aligned}
& \sum_{j=0}^{k} b_{j}\left[\psi_{i}^{k-j+1}-\psi_{i}^{k-j}\right] \\
& \quad+r\left[\psi_{i-2}^{k+1}-4 \psi_{i-1}^{k+1}+6 \psi_{i}^{k+1}-4 \psi_{i+1}^{k+1}+\psi_{i+2}^{k+1}\right] \\
& \quad=d_{i}^{k+1} .
\end{aligned}
$$

Since the points are at $i=1$ and $i=M-1$, we need points outside the domain associated with the calculation. We apply boundary conditions $\frac{\partial^{2} \psi(0, t)}{\partial x^{2}}=$ $\eta_{1}(t)$ and $\frac{\partial^{2} \psi(L, t)}{\partial x^{2}}=\eta_{2}(t)$ to overcome this problem. Finally, these relations can be seen in matrix form of system (Eq. (11)) as follows:

$$
\left\{\begin{array}{l}
\mathbf{A} \boldsymbol{\Psi}^{1}=\boldsymbol{\Psi}^{0}+\mathbf{d}^{1}, \\
\mathbf{A} \Psi^{k+1}=\sum_{j=0}^{k-1}\left(b_{j}-b_{j+1}\right) \boldsymbol{\Psi}^{k-j}+b_{k} \Psi^{0}+\mathbf{d}^{k+1}, \\
\Psi^{0}=\mathbf{\Phi}
\end{array}\right.
$$

where:

$$
\begin{gathered}
\boldsymbol{\Psi}^{k}=\left[\begin{array}{c}
\psi_{0}^{k} \\
\psi_{1}^{k} \\
\psi_{2}^{k} \\
\vdots \\
\psi_{M-1}^{k} \\
\psi_{M}^{k}
\end{array}\right], \quad \mathbf{d}^{k}=\left[\begin{array}{c}
0 \\
r_{1} g_{1}^{k}-r h^{2} \eta_{1}^{k} \\
r_{1} g_{2}^{k} \\
\vdots \\
r_{1} g_{M-2}^{k} \\
r_{1} g_{M-1}^{k}-r h^{2} \eta_{M-1}^{k} \\
0 \\
\chi(0) \\
\chi(h) \\
\chi(2 h) \\
\vdots \\
\chi((M-1) h) \\
\chi(M h)
\end{array}\right],\left[\begin{array}{c}
0 \\
\end{array}\right]
\end{gathered}
$$

and $\mathbf{A}$ is the coefficients matrix where it is defined as shown in Box I.

Theorem 1. Let $\psi_{i}^{k}(1 \leq i \leq M, k \geq 1)$ be the numerical solution of Eq. (1), then the difference scheme considered here is uniquely solvable.

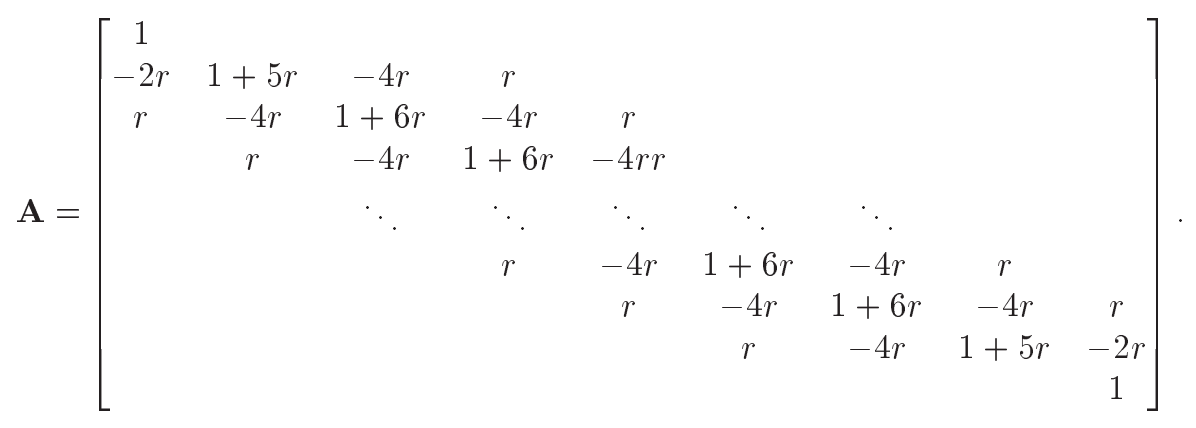


Proof. Eq. (11) together with boundary conditions $\psi_{0}^{k}=0$ and $\psi_{M}^{k}=0$ will construct a penta-diagonal matrix system whose coefficients' matrix is positively definite and invertible. After that, the related matrix system can be solved iteratively. In this regard, initial vector $\Psi^{0}$ must be calculated using the initial condition of Problem (1). Hence, the difference scheme is uniquely solvable and this completes the proof.

\subsection{The physical understanding of the fractional derivative}

Now, we consider an arbitrary plane with fractal structure [5]. As we know, the shortest path between two different points on the plane, namely $X$ and $Y$, is not a line, and consequently, the actual distance $\left(d s_{E}\right)$ between $X$ and $Y$ can be presented in the following form:

$$
d s_{E}=c d s^{\beta},
$$

where $\beta$ is the finite dimension and $c$ is an arbitrary constant. In [5], the author showed that the projection of $d s_{E}$ into the horizontal axis implies the Cantor sets, and the length has the following form:

$$
\Delta_{t} X Y=c d t^{\beta} \text {. }
$$

On the other hand, Eq. (14) has the following transform:

$$
s_{E}=c s^{\beta} \text {. }
$$

\section{Error analysis}

Hereunder, we consider the difference form of equation defined in Eq. (11) and estimate the order of accuracy or the rate of convergence as $O\left(\tau^{2-\alpha}, h^{2}\right)$. So, the consistency of our scheme is obtainable.

Theorem 2. The numerical scheme, considered here, has $(2-\alpha)$-order accuracy in time dimension and second-order accuracy in space dimension.

Proof. By using the Taylor expanding on Eq. (11) around $\left(x_{i}, t_{k+1}\right)$ and summarizing the formula, the following relations can be obtained:

$$
\begin{aligned}
\sum_{j=0}^{k} b_{j} & \left\{\left(\psi_{i}^{k+1}-\left.j \tau \frac{\partial \psi}{\partial t}\right|_{i} ^{k+1}+\left.\frac{j^{2} \tau^{2}}{2 !} \frac{\partial^{2} \psi}{\partial t^{2}}\right|_{i} ^{k+1}+\cdots\right)\right. \\
& -\left(\psi_{i}^{k+1}-\left.(j+1) \tau \frac{\partial \psi}{\partial t}\right|_{i} ^{k+1}\right. \\
+ & \left.\left.\left.\frac{(j+1)^{2} \tau^{2}}{2 !} \frac{\partial^{2} \psi}{\partial t^{2}}\right|_{i} ^{k+1}+\cdots\right)\right\} \\
+ & r\left\{\left(\psi_{i}^{k+1}+\left.2 h \frac{\partial \psi}{\partial x}\right|_{i} ^{k+1}+\left.\frac{4 h^{2}}{2 !} \frac{\partial^{2} \psi}{\partial x^{2}}\right|_{i} ^{k+1}\right.\right.
\end{aligned}
$$

$$
\begin{aligned}
& \left.+\left.\frac{8 h^{3}}{3 !} \frac{\partial^{3} \psi}{\partial x^{3}}\right|_{i} ^{k+1}+\cdots\right) \\
& -4\left(\psi_{i}^{k+1}+\left.h \frac{\partial \psi}{\partial x}\right|_{i} ^{k+1}+\left.\frac{h^{2}}{2 !} \frac{\partial^{2} \psi}{\partial x^{2}}\right|_{i} ^{k+1}+\cdots\right) \\
& +6 \psi_{i}^{k+1}-4\left(\psi_{i}^{k+1}-\left.h \frac{\partial \psi}{\partial x}\right|_{i} ^{k+1}+\left.\frac{h^{2}}{2 !} \frac{\partial^{2} \psi}{\partial x^{2}}\right|_{i} ^{k+1}\right. \\
& \left.-\left.\frac{h^{3}}{3 !} \frac{\partial^{3} \psi}{\partial x^{3}}\right|_{i} ^{k+1}+\cdots\right) \\
& +\left(\psi_{i}^{k+1}-\left.2 h \frac{\partial \psi}{\partial x}\right|_{i} ^{k+1}+\left.\frac{4 h^{2}}{2 !} \frac{\partial^{2} \psi}{\partial x^{2}}\right|_{i} ^{k+1}\right. \\
& \left.\left.-\left.\frac{8 h^{3}}{3 !} \frac{\partial^{3} \psi}{\partial x^{3}}\right|_{i} ^{k+1}+\cdots\right)\right\}=d_{i}^{k+1} \cdot
\end{aligned}
$$

According to Expressions (8)-(10), Eq. (17) can be rewritten in this form:

$$
\begin{aligned}
\tau^{\alpha} \Gamma(2-\alpha)\{ & \left.\frac{\partial^{\alpha} \psi}{\partial t^{\alpha}}\right|_{i} ^{k+1}+\left.\beta \frac{\partial^{4} \psi}{\partial x^{4}}\right|_{i} ^{k+1}-g_{i}^{k+1} \\
& +\left.\frac{\tau^{2-\alpha}}{\Gamma(2-\alpha)} \frac{\partial^{2} \psi}{\partial t^{2}}\right|_{i} ^{k+1} \sum_{j=0}^{k} b_{j} \frac{2 j+1}{2 !} \\
& \left.-\left.\frac{h^{2}}{6} \frac{\partial^{6} \psi}{\partial x^{6}}\right|_{i} ^{k+1}+\cdots\right\}=0 .
\end{aligned}
$$

Consequently:

$$
\frac{\partial^{\alpha} \psi(x, t)}{\partial t^{\alpha}}+\beta \frac{\partial^{4} \psi(x, t)}{\partial x^{4}}-g(x, t)+O\left(\tau^{2-\alpha}, h^{2}\right)=0
$$

On the other hand, the order of accuracy of our suggested scheme is $O\left(\tau^{2-\alpha}, h^{2}\right)$, and the difference equation (Eq. (11)) is consistent with partial differential equation (Eq. (1)).

\subsection{Stability analysis}

Now, the stability of our scheme is investigated by using the properties of the Fourier stability analysis. We remind that in differential equations, a reliable analytical tool to check the stability of finite difference approximation is the Fourier stability analysis.

Lemma 1. The stability analysis of our scheme is independent of the source term $g(x, t)$.

Proof. Let $\psi\left(x_{i}, t_{k}\right)$ and $\psi_{i}^{k}$ be the exact and numerical solutions to the proposed problem, respectively. Then, the approximate equation has the following form: 


$$
\frac{\partial^{\alpha} \psi^{k+1}(x, t)}{\partial t^{\alpha}}+\beta \frac{\partial^{4} \psi^{k+1}(x, t)}{\partial x^{4}}=g(x, t)
$$

where substituting Eqs. (8) and (9) into Eq. (20) implies that:

$$
\begin{gathered}
\frac{\tau^{-\alpha}}{\Gamma(2-\alpha)} \sum_{j=0}^{k} b_{j}\left[\psi_{i}^{k-j+1}-\psi_{i}^{k-j}\right]+\frac{\beta}{h^{4}}\left[\psi_{i-2}^{k+1}\right. \\
\left.-4 \psi_{i-1}^{k+1}+6 \psi_{i}^{k+1}-4 \psi_{i+1}^{k+1}+\psi_{i+2}^{k+1}\right] \\
+O\left(h^{2}+\tau^{2-\alpha}\right)=g\left(x_{i}, t_{k+1}\right)
\end{gathered}
$$

Assuming $e_{i}^{k}=\psi\left(x_{i}, t_{k}\right)-\psi_{i}^{k}$, one will set:

$$
\begin{aligned}
& \frac{\tau^{-\alpha}}{\Gamma(2-\alpha)} \sum_{j=0}^{k} b_{j}\left[\psi\left(x_{i}, t_{k-j+1}\right)-\psi\left(x_{i}, t_{k-j}\right)\right] \\
& \quad+\frac{\beta}{h^{4}}\left[\psi\left(x_{i-2}, t_{k+1}\right)-4 \psi\left(x_{i-1}, t_{k+1}\right)\right. \\
& \left.\quad+\psi\left(x_{i}, t_{k+1}\right)-4 \psi\left(x_{i+1}, t_{k+1}\right)+\psi\left(x_{i+2}, t_{k+1}\right)\right] \\
& \quad+O\left(h^{2}+\tau^{2-\alpha}\right)=\frac{\tau^{-\alpha}}{\Gamma(2-\alpha)} \sum_{j=0}^{k} b_{j}\left[e_{i}^{k-j+1}-e_{i}^{k-j}\right] \\
& \quad+\frac{\beta}{h^{4}}\left[e_{i-2}^{k+1}-4 e_{i-1}^{k+1}+6 e_{i}^{k+1}-4 e_{i+1}^{k+1}+e_{i+2}^{k+1}\right] \\
& \quad+g\left(x_{i}, t_{k+1}\right),
\end{aligned}
$$

and therefore:

$$
\begin{aligned}
\frac{\tau^{-\alpha}}{\Gamma(2-\alpha)} \sum_{j=0}^{k} b_{j}\left[e_{i}^{k-j+1}-e_{i}^{k-j}\right]+\frac{\beta}{h^{4}}\left[e_{i-2}^{k+1}\right. \\
\left.-4 e_{i-1}^{k+1}+6 e_{i}^{k+1}-4 e_{i+1}^{k+1}+e_{i+2}^{k+1}\right]=0 .
\end{aligned}
$$

So, the scheme's stability is independent of source term $g$ and the proof is completed. For more details, one can refer to $[30,31]$.

According to the Lemma 1 , to investigate the stability analysis, we can suppose that $g=0$. By applying $e_{j}^{k}$ defined in Lemma 1 , we can rewrite Eq. (23) as follows:

$$
\begin{gathered}
\sum_{j=0}^{k} b_{j}\left[e_{i}^{k-j+1}-e_{i}^{k-j}\right]+r\left[e_{i-2}^{k+1}-4 e_{i-1}^{k+1}+6 e_{i}^{k+1}\right. \\
\left.-4 e_{i+1}^{k+1}+e_{i+2}^{k+1}\right]=0
\end{gathered}
$$

and therefore, by replacing $i$ to $s$ :

$$
\begin{gathered}
r e_{s-2}^{k+1}-4 r e_{s-1}^{k+1}+(1+6 r) e_{s}^{k+1}-4 r e_{s+1}^{k+1}+r e_{s+2}^{k+1} \\
=b_{k} e_{s}^{0}+\sum_{j=0}^{k-1}\left(b_{j}-b_{j+1}\right) e_{s}^{k-j}
\end{gathered}
$$

Error $e_{s}^{k}$ can be described by a discrete Fourier series $e_{s}^{k}=\sum_{q} \epsilon_{q}^{k} e^{i q s h}$, where $q$ is the spatial wave number supported by lattice, $\epsilon$ is the time dependence of the solution, and $s h$ is the position along the grid [31]. We also assume that the coefficients of the equation are so slowly changing that they can be considered constant in both space and time dimensions.

The solution is stable in time if we have $|\epsilon| \leq 1$. Otherwise, since $k$ is a positive integer, $\epsilon^{k}$ will be a quickly growing amount and corresponding to it, the error will increase (see [31]). So, we assay that under what circumstances $|\epsilon| \leq 1$.

Substituting general mode $e_{s}^{k}=\epsilon_{q}^{k} e^{i q s h}$ into difference form (Eq. (25)) leads to:

$$
\begin{aligned}
\epsilon_{q}^{k+1} & {\left[1+32 r \sin ^{2}\left(\frac{q h}{2}\right) \sin ^{2}\left(\frac{q h}{4}\right)\right] } \\
= & \sum_{j=0}^{k}\left(b_{j}-b_{j+1}\right) \epsilon_{q}^{k-j} .
\end{aligned}
$$

Theorem 3. The finite difference approximation obtained in Eq. (11) is unconditionally stable.

Proof. According to Eq. (26) and that $\mid 1+32 r \sin ^{2}$ $\left(\frac{q h}{2}\right) \sin ^{2}\left(\frac{q h}{4}\right) \mid \geq 1$, these relations are true:

$$
\begin{aligned}
\left|\epsilon_{q}^{k+1}\right| & \leq \sum_{j=0}^{k}\left(b_{j}-b_{j+1}\right)\left|\epsilon_{q}^{k-j}\right| \leq\left|\epsilon_{q}^{\{k\}}\right|_{\max } \sum_{j=0}^{k}\left(b_{j}-b_{j+1}\right) \\
& =\left|\epsilon_{q}^{\{k\}}\right|_{\max }\left(b_{0}-b_{k+1}\right)<\left|\epsilon_{q}^{\{k\}}\right|_{\max }
\end{aligned}
$$

where:

$$
\left|\epsilon_{q}^{\{k\}}\right|_{\max }=\max \left\{\left|\epsilon_{q}^{0}\right|,\left|\epsilon_{q}^{1}\right|,\left|\epsilon_{q}^{2}\right|, \cdots,\left|\epsilon_{q}^{k}\right|\right\} .
$$

Thus:

$$
\left|\epsilon_{q}^{k+1}\right|<\left|\epsilon_{q}^{0}\right|
$$

Now, by using the Parseval's relation [31], $\left\|e^{k}\right\|_{2}^{2}=$ $\sum_{q}\left|\epsilon_{q}^{k}\right|^{2}$; therefore, stability condition (29) can be rewritten as:

$$
\left\|e^{k}\right\|_{2}<\left\|e^{0}\right\|_{2}
$$

So, the perturbation of the general mode in any time level, $k$, does not grow and is smaller than or equal to its initial perturbation, i.e. our finite difference approximation is unconditionally stable. This completes the proof.

\subsection{Convergence analysis}

Two important conditions that are usually discussed 
in finite difference approximations are consistency and stability analyses. Furthermore, we know that the convergence of a fractional difference method can be obtained using its consistency and stability under relatively weak conditions. Hereunder, we check the convergence condition of the suggested method.

Theorem 4. The solution of finite difference approximation (Eq. (11)) is unconditionally convergent with the exact solution of Problem (1).

Proof. According to Eq. (12), we consider the homogeneous case in the following form:

$$
\left\{\begin{array}{l}
\Psi^{1}=\mathbf{A}^{-1} \Psi^{0} \\
\Psi^{k+1}=\sum_{j=0}^{k-1}\left(b_{j}-b_{j+1}\right) \mathbf{A}^{-1} \Psi^{k-j}+b_{k} \mathbf{A}^{-1} \Psi^{0} \\
\Psi^{0}=\boldsymbol{\Phi}
\end{array}\right.
$$

Substituting error vector $\mathbf{e}^{k}=\left[e_{1}^{k}, e_{2}^{k}, \cdots, e_{M-1}^{k}\right]^{T}$ into this matrix system, we obtain:

$$
\begin{aligned}
\mathbf{e}^{k+1}= & \left(b_{0}-b_{1}\right) \mathbf{A}^{-\mathbf{1}} \mathbf{e}^{k}+\left(b_{1}-b_{2}\right) \mathbf{A}^{-\mathbf{1}} \mathbf{e}^{k-1}+\cdots \\
& +\left(b_{k-1}-b_{k}\right) \mathbf{A}^{-\mathbf{1}} \mathbf{e}^{1}+b_{k} \mathbf{A}^{-\mathbf{1}} \mathbf{e}^{0}+\mathbf{A}^{-\mathbf{1}} \mathbf{R}^{k+1},
\end{aligned}
$$

where $\mathbf{R}^{k}$ is the error vector obtained from the difference scheme. By applying the property of 2-norm, the following results can be obtained:

$$
\begin{aligned}
\left\|\mathbf{e}^{k+1}\right\|_{2} & \leq\left(b_{0}-b_{1}\right)\left\|\mathbf{A}^{-\mathbf{1}}\right\|_{2}\left\|\mathbf{e}^{k}\right\|_{2} \\
& +\left(b_{1}-b_{2}\right)\left\|\mathbf{A}^{-\mathbf{1}}\right\|_{2}\left\|\mathbf{e}^{k-1}\right\|_{2}+\cdots \\
& +\left(b_{k-1}-b_{k}\right)\left\|\mathbf{A}^{-\mathbf{1}}\right\|_{2}\left\|\mathbf{e}^{1}\right\|_{2} \\
& +b_{k}\left\|\mathbf{A}^{-\mathbf{1}}\right\|_{2}\left\|\mathbf{e}^{0}\right\|_{2}+\left\|\mathbf{A}^{-\mathbf{1}}\right\|_{2}\left\|\mathbf{R}^{k+1}\right\|_{2} \\
& \leq\left(b_{0}-b_{1}\right)\left\|\mathbf{A}^{-\mathbf{1}}\right\|_{2}\left\|\mathbf{e}^{\{k\}}\right\|_{2} \\
& +\left(b_{1}-b_{2}\right)\left\|\mathbf{A}^{-\mathbf{1}}\right\|_{2}\left\|\mathbf{e}^{\{k\}}\right\|_{2}+\cdots \\
& +\left(b_{k-1}-b_{k}\right)\left\|\mathbf{A}^{-\mathbf{1}}\right\|_{2}\left\|\mathbf{e}^{\{k\}}\right\|_{2} \\
& +b_{k}\left\|\mathbf{A}^{-\mathbf{1}}\right\|_{2}\left\|\mathbf{e}^{\{k\}}\right\|_{2}+\left\|\mathbf{A}^{-\mathbf{1}}\right\|_{2}\left\|\mathbf{R}^{k+1}\right\|_{2} \\
& =\left\|\mathbf{A}^{-\mathbf{1}}\right\|_{2}\left\|\mathbf{e}^{\{k\}}\right\|_{2}\left\{\left(b_{0}-b_{1}\right)+\left(b_{1}-b_{2}\right)\right. \\
& \left.+\cdots+\left(b_{k-1}-b_{k}\right)+b_{k}\right\}+\left\|\mathbf{A}^{-\mathbf{1}}\right\|_{2}\left\|\mathbf{R}^{k+1}\right\|_{2}
\end{aligned}
$$

$$
\leq\left\|\mathbf{A}^{-1}\right\|_{2}\left\|\mathbf{e}^{\{k\}}\right\|_{2}+\left\|\mathbf{A}^{-1}\right\|_{2}\left\|\mathbf{R}^{k+1}\right\|_{2}
$$

and thus:

$$
\left\|\mathbf{e}^{k+1}\right\|_{2} \leq\left\|\mathbf{A}^{-1}\right\|_{2}\left\|e^{0}\right\|_{2}+\left\|\mathbf{A}^{-1}\right\|_{2}\left\|\mathbf{R}^{k+1}\right\|_{2}
$$

Since the method is consistent, $\left\|\mathbf{R}^{\mathbf{k}+\mathbf{1}}\right\|_{2} \rightarrow 0$; while $\left\|\mathbf{A}^{-1}\right\|_{2}$ is bounded, then our scheme implies that $\left\|\mathbf{e}^{\mathbf{k}+\mathbf{1}}\right\|_{2} \rightarrow 0$, i.e. the method is convergent. This proves the theorem.

\section{Numerical experiments}

In this section, we present a series of numerical tests and provide some results to confirm our theoretical results. Our fundamental objectives are to investigate the convergence analysis of the numerical solutions with respect to $\tau$ and $h$, the temporal and spatial stepsize, respectively. Therefore, to measure the accuracy, we compute errors $\left\|\psi\left(x_{i}, t_{k}\right)-\psi_{i}^{k}\right\|$ in two norms: $L_{2}$ and $L_{\infty}$. Modeling this example efficiently results in the success of our numerical method. We have evaluated the numerical results at $T=1$.

Example. Let us consider a compact fourth-order subdiffusion equation as in the following problem.

$$
\left\{\begin{array}{l}
\frac{\partial^{\alpha} \psi(x, t)}{\partial t^{\alpha}}=\frac{\partial^{4} \psi(x, t)}{\partial x^{4}}+f(x, t) \\
\quad x \in I=[0,1], \quad t \geq 0, \quad 0<\alpha<1 \\
\psi(0, t)=\psi(1, t)=0 \\
\quad t \geq 0 \\
\frac{\partial^{2} \psi(0, t)}{\partial x^{2}}=\frac{\partial^{2} \psi(1, t)}{\partial x^{2}}=0 \\
t \geq 0
\end{array}\right.
$$

where the corresponding force term is:

$$
f(x, t)=\frac{2 t^{2-\alpha}}{\Gamma(3-\alpha)} \sin (2 \pi x)+16 \pi^{4} t^{2} \sin (2 \pi x),
$$

with the initial condition:

$$
\psi(x, 0)=0,
$$

where the exact solution to Eqs. (34) and (35) is $\psi(x, t)=t^{2} \sin (2 \pi x)$.

For the convenience of calculations, two figures are presented. Figure 1(a) shows the surface of the numerical solution obtained from the proposed scheme; in Figure 1(b), we denote $\alpha=0.5$ and $h=\tau=0.025$, then the values of numerical solutions are plotted for $t=0,0.3,0.5,0.7,1$. In Figure $2(\mathrm{a})$ and (b), the nodal values of the exact and numerical solutions have been compared for two states, when $x=0.3$ and $x=0.7$ for various $t$. These figures show that the yielding solutions by the new method are in high agreement with the exact solutions. 


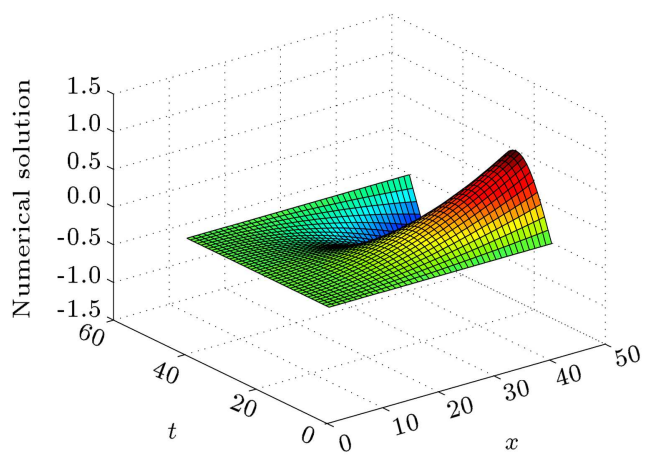

(a)

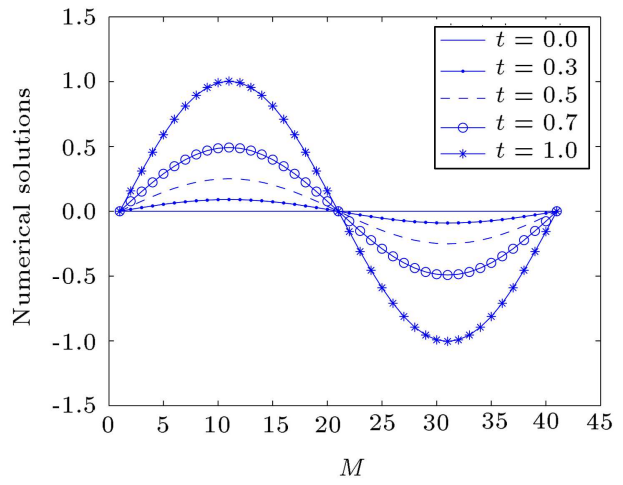

(b)

Figure 1. Numerical solutions of Eqs. (34) and (35): (a) For $x=0.5$ and $N=40$ at different time levels, (b) surface of the numerical solution for $M=N=40$ and $\alpha=0.5$.

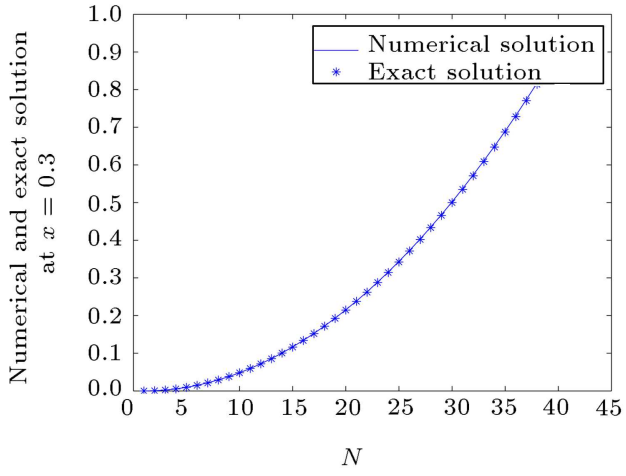

(a)

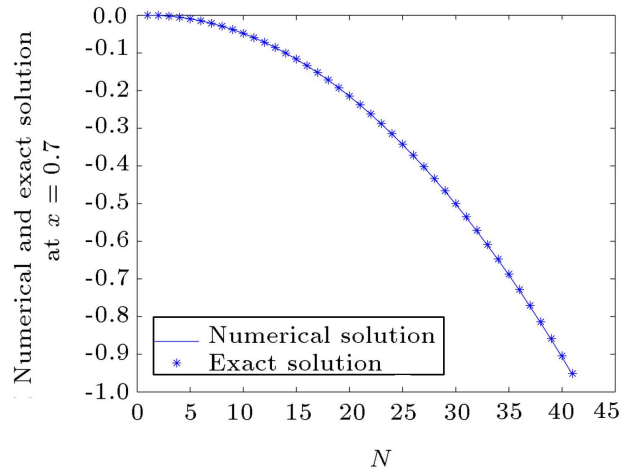

(b)

Figure 2. Comparison of the exact and numerical solutions of Eqs. (34) and (35): (a) For $x=0.3$ and $N=40$ at different time levels, and (b) for $x=0.7$ and $N=40$ at different time levels.

\section{Conclusions}

In this paper, we consider a compact class of fourthorder subdiffusion equations as an initial-boundary value problem and solve it numerically. We presented an implicit finite difference scheme to estimate the numerical solution. To discretize the suggested problem, we used a backward difference formula for the fractional derivative in time dimension and a centered difference formula for the space dimension derivative. The obtained results showed that the proposed scheme requires only small evaluations, and then provides accurate solutions. Furthermore, our scheme is rapidly convergent and it provides numerical solutions in good agreement with exact ones. The numerical solutions were obtained using Matlab.

\section{References}

1. He, J.H. "A tutorial review on fractal space time and fractional calculus", International J. Theor. Phys., 53(11), pp. 3698-3718 (2014).

2. Zhu, W.H., Pan, Y.Y., Li, Z.B. and Wang, Q.L. "One dimensional heat conduction equation of the polar bear hair", Thermal Scie., 19, pp. 179-181 (2015).

3. He, J.H. "Some applications of nonlinear fractional differential equations and their applications", Bull. Sci. Technol., 15(2), pp. 86-90 (1999).

4. Liu, H.Y., He, J.H. and Li, Z.B. "Fractional calculus for nanoscale flow and heat transfer", Int. J. Numer. Method Heat Fluid Flow, 24(6), pp. 1227-1250 (2014).

5. He, J.H. "String theory in a scale dependent discontinuous space-time", Chaos Solit. Fract., 36(3), pp. 542545 (2008).

6. Sayevand, K. and Pichaghchi, K. "Successive approximation: A survey on stable manifold of fractional differential systems", Frac. Calculus and Appl. Anal., 18(3), pp. 621-641 (2015).

7. Sayevand, K. "Analytical treatment of Volterra integro-differential equations of fractional order", Appl. Math. Modell., 39(15), pp. 4330-4336 (2015).

8. Podlubny, I., Fractional Differential Equations, Academic Press, San Diego (1999).

9. Oldham, K.B. and Spanier, J., The Fractional Calculus, New York, Academic Press (1974).

10. Metzler, R. and Klafter, J. "The restaurant at the end of the random walk: Recent developments in fractional 
dynamics of anomalous transport processes", J. Phys. A, 37, pp. 161-208 (2004).

11. Sunil, K. "A numerical study for solution of time fractional nonlinear shallow-water equation in oceans", Z. Naturforsch. A, 68, pp. 1-7 (2013).

12. Sunil, K. "Numerical computation of time-fractional FokkerPlanck equation arising in solid state physics and circuit theory", Z. Naturforsch. A, 68, pp. 1-8 (2013).

13. Sunil, K. "A new analytical modelling for telegraph equation via Laplace transform", Appl. Math. Modell., 38(13), pp. 3154-3163 (2014).

14. Sunil, K. and Rashidi, M.M. "New analytical method for gas dynamics equation arising in shock fronts", Compu. Phys. Communi., 185(7), pp. 1947-1954 (2014).

15. He, J.H. "Homotopy perturbation technique", Comput. Methdos Appl. Mech. Engrg., 178, pp. 257-262 (1999).

16. He, J.H. "Homotopy perturbation method: A new nonlinear analytical technique", Appl. Math. Comput., 135, pp. 73-79 (2003).

17. He, J.H. "Variational iteration method - Some recent results and new interpretations", J. Comput. Appl. Math., 207, pp. 3-17 (2007).

18. He, J.H. and Wu, X.H. "Exp-function method for nonlinear wave equations", Chaos Soliton. Fract., 30, pp. 700-708 (2006).

19. He, J.H., Elagan, S.K. and Li, Z.B. "Geometrical explanation of the fractional complex transform and derivative chain rule for fractional calculus", Phys. Lett. A, 376(4), pp. 257-259 (2012).

20. Klages, R., Radons, G. and Sokolov, I.M., Anomalous Transport: Foundations and Applications, WileyVCH, Weinheim (2008).

21. Gorenflo, R., Mainardi, F., Moretti, D. and Pagnini, G. "Time-fractional diffusion: A discrete random walk approach", Nonlinear Dynamics, 29, pp. 129-143 (2002).

22. Metzler, R. and Klafter, J. "The random walks guide to anomalous diffusion: a fractional dynamics approach", Phys. Reports, 339(1), pp. 1-77 (2000).

23. Yuste, S.B. and Lindenberg, K. "Trapping reactions with sub-diffusive traps and particles characterized by different anomalous diffusion exponents", Phys. Rev. E, 72, pp. 061-103 (2005).

24. Mainardi, F. "Fractional diffusive waves in viscoelastic solids", Nonlinear Waves in Solids, ASME/AMR, Fairfield, pp. 93-97 (1995).
25. Wang, H., Wang, K. and Sircar, T. "A direct $\mathrm{O}(\mathrm{N}$ $\log (2 \mathrm{~N}))$ finite difference method for fractional diffusion equations", J. Comput. Phys., 229, pp. 8095-8104 (2010).

26. Yuste, S.B. and Acedo, L. "An explicit finite difference method and a new Von Neumann-type stability analysis for fractional diffusion equations", Physica, A, pp. 326-334 (2004).

27. Zhuang, P., Liu, F., Anh, V. and Turner, I. "New solution and analytical techniques of the implicit numerical method for the anomalous sub-diffusion equation", SIAM Journal of Num. Anal., 46(2), pp. 1079-1095 (2008).

28. Liu, F., Zhuang, P., Anh, V., Turner, I. and Burrage, K. "Stability and convergence of the difference methods for the space-time fractional advection-diffusion equation", Appl. Math. Comput., 191, pp. 12-20 (2007).

29. Mainardi, F. "Fractional calculus in wave propagation problems", Math. Phys., 19, pp. 20-52 (2012).

30. Chalabi, A. "Stable upwind schemes for hyperbolic conservation laws with source terms", IMA Journal of Num. Anal., 12, pp. 217-241 (1992).

31. Yuste, S.B. and Quintana-Murillo, J. "A finite difference method with non-uniform time steps for fractional diffusion equations", Comp. Phys. Communications, 183, pp. 2594-2600 (2012).

\section{Biographies}

Khosro Sayevand is an Associate Professor at Malayer University. He has published more than 40 articles in ISI-listed journals. His current research interests mainly cover fractional calculus and perturbation theory. He received his BS degree from the University of Mazandaran, Iran, in 1997, his MS degree from Amirkabir University of Technology, Tehran, Iran, in 2000, and his PhD degree from the Iran, University of Science and Technology, Iran, in 2010, all in Applied Mathematics.

Fateme Arjang received his BS and MS degrees in Mathematics from Shahrood University and Mazandaran University, Iran, in 2006 and 2008, respectively. Her research interests include numerical analysis. At present, she is a PhD student at Malayer University. 